\title{
COMPUTATIONS OF THE YAMABE INVARIANT
}

\author{
JIMMY PETEAN
}

\begin{abstract}
For a compact connected manifold $M$ of dimension $n \geq 4$ with no metric of positive scalar curvature, we prove that the Yamabe invariant is unchanged under surgery on spheres of dimension different from $1, n-2$ and $n-1$. We use this result to give new exact computations of the Yamabe invariant in dimension four and display new examples of compact four-manifolds which do not admit Einstein metrics.
\end{abstract}

\section{Introduction}

Consider a smooth compact connected manifold $M^{n}$ of dimension $n$. It is well known that set of the critical points of the functional

$$
\mathcal{S}(g)=\frac{\int_{M} s_{g} d \operatorname{vol}_{g}}{\left(\operatorname{Vol}_{g}(M)\right)^{\frac{n-2}{n}}},
$$

(on the space $\mathcal{M}$ of all Riemannian metrics on $M$ ) is the set of Einstein metrics on $M$. But it is also well known that this functional is never bounded below or above and hence it is not possible to prove existence of critical points by minimizing or maximizing the functional. An alternative strategy to find critical points is a minimax argument, the first ideas of which were introduced by Yamabe in [15]. First we have to restrict the functional to some fixed conformal class; the functional is bounded below in this subspace and we can define the Yamabe constant of the conformal class $\mathcal{C}$ by

$$
Y(M, \mathcal{C})=\inf _{g \in \mathcal{C}} \mathcal{S}(g)
$$

Moreover, the infimum is always achieved by a smooth metric, which therefore has constant scalar curvature. Yamabe claimed to prove this result in [15], but his proof contained an error. This was recognized by Trudinger (see [13]), and the problem was finally solved in several steps by Trudinger [13], Aubin [3] and Schoen [11].

We now define the Yamabe invariant of $M$ by

$$
Y(M)=\sup _{\mathcal{C}} Y(M, \mathcal{C})
$$

(where the supremum is taken over the family of all conformal classes of metrics on $M)$.

Received July 17, 1998. 
This invariant was introduced by O. Kobayashi in [5] and it is also frequently called the sigma constant of $M$ [12]. Note that the Yamabe invariant of $M$ is an invariant of the smooth structure of $M$.

We will be concerned in this paper with manifolds for which the Yamabe invariant is non-positive. This is equivalent to say that the manifold does not admit any metric of strictly positive scalar curvature. In this case, there is always a unique metric of constant scalar curvature and unit volume in each conformal class of metrics on $M$, and the Yamabe invariant is the supremum of the scalar curvature over the space of metrics of constant scalar curvature and unit volume. Furthermore, if the Yamabe invariant is realized by such a metric, then the metric is a critical point of the functional $\mathcal{S}$ and therefore it is an Einstein metric.

This is the minimax approach to find Einstein metrics we mentioned in the beginning of this introduction. For the proofs of these last statements, and additional references, see [12, Section 1$]$.

Recall also that, still assuming that $M$ does not admit any metric of positive scalar curvature,

$$
Y(M)=-\left(\inf _{\mathcal{M}} \int_{M}\left|s_{g}\right|^{n / 2} d \operatorname{vol}_{g}\right)^{2 / n},
$$

where $\mathcal{M}$ is, as before, the space of all Riemannian metrics on $M$ (see $[2,6]$ ). Hence all our results regarding the Yamabe invariant of a manifold can also be reinterpreted in terms of the infimum over $\mathcal{M}$ of the $L^{n / 2}$ norm of the scalar curvature.

We will prove the following theorem:

Theorem 1. Suppose $M$ is a connected smooth compact manifold of dimension $n \geq 4$ with $Y(M) \leq 0$. Let $\widehat{M}$ be a manifold obtained from $M$ by performing surgeries on spheres of dimension different from 1, n-1 and n-2. Then $Y(\widehat{M})=$ $Y(M)$.

The proof of Theorem 1 will be based on the results of [8], where it is proved that surgery in codimension greater than 2 does not decrease the Yamabe invariant (assuming that the invariant is non-positive).

Some computations of the invariant have been carried out in low dimensions. In dimension four, LeBrun computed the Yamabe invariant of all compact complex surfaces of Kähler type which do not admit metrics of positive scalar curvature [6]. We will use Theorem 1 and the computations in [6] to give new exact computations of the Yamabe invariant for four-dimensional manifolds. Note that the computation of the invariant in dimension two follows from the GaussBonnet formula and in dimension three, in the non-positive case, would follow from Anderson's program for the hyperbolization conjecture [1].

We will also show that it follows from Theorem 1 that the Yamabe invariant of a manifold of dimension greater than four is unchanged under connecting 
sum with the product of two spheres (always assuming that the invariant is non-positive).

Finally, we will use these results to display new examples of compact fourdimensional manifolds which do not admit Einstein metrics. For instance, we will prove:

Theorem 2. The connected sum of a compact complex hyperbolic four-manifold with any number of copies of $S^{1} \times S^{3}$ does not admit any Einstein metric.

Note that similar results, in the case of real hyperbolic four-dimensional manifolds, have been proved by Sambusetti in [9].

Numerous other examples will be given in the last section of this paper.

\section{Computations of the invariant}

We will now prove Theorem 1 . We will use a "double surgery" argument and the following result, proved in $[8$, Theorem 1$]$ :

Proposition 1. If $\widehat{M}$ is obtained from $M$ by performing surgery in codimension at least 3 and $Y(M) \leq 0$, then $Y(\widehat{M}) \geq Y(M)$.

Since we know that, under certain restrictions in codimension, surgery can only increase the Yamabe invariant, what we will do is to try to kill the surgery we realized by another surgery that also verifies the restriction in codimension.

There is a canonical way to undo a surgery done on a manifold. Let $\mathcal{S}^{k}$ be a $k$-dimensional sphere embedded in the manifold $M$ with trivialized normal bundle. Let $\widehat{M}$ be the manifold obtained by doing surgery on $\mathcal{S}^{k}$ (of course, $\widehat{M}$ will depend on the homotopy class of the trivialization of the normal bundle). The boundary of a tubular neighborhood $U$ of $\mathcal{S}^{k}$ is diffeomorphic to $S^{k} \times S^{n-k-1}$ and $\widehat{M}$ is obtained by gluing $D^{k+1} \times S^{n-k-1}$ to $M-U$ along their boundaries. Doing surgery on the $(n-k-1)$-belt sphere, $\{0\} \times S^{n-k-1} \subset D^{k+1} \times S^{n-k-1}$, in $\widehat{M}$, obviously produces a manifold diffeomorphic to $M$.

Assume now that $k \leq n-3$, and $n-k-1 \leq n-3$. Then, following from Theorem 2 and previous observations, $Y(M) \leq Y(\widehat{M}) \leq Y(M)$. Therefore, $Y(M)=Y(\widehat{M})$. Note that $\widehat{M}$ does not admit any metric of positive scalar curvature because if that were the case then $M$ would also admit such a metric (since the family of manifolds which admit metrics of positive scalar curvature is closed under surgery on codimension greater than two from the results of Gromov-Lawson and Schoen-Yau; see $[4,10])$.

The previous condition on $k$ can also be written as $2 \leq k \leq n-3$. To conclude the proof of Theorem 1 we therefore need only to deal with the case $k=0$. There is another canonical way to undo surgery in the 0 -dimensional case. Doing 0-dimensional surgery on a connected manifold $M$ means that we take out 2 disjoint balls in $M$ and we glue the boundaries to the boundary of $S^{n-1} \times[0,1]$. Pick any point $q \in S^{n-1}$ and consider the loop obtained by joinning the endpoints of $\{q\} \times[0,1]$ with a smoothly embedded curve inside 
of $M$. The result will be a smoothly embedded circle in the manifold obtained by doing the 0 -dimensional surgery on $M$. Doing surgery on such a circle will undo the 0-dimensional surgery. Another way to see this is the following: the manifold obtained by doing 0-dimensional surgery on $M$ is $M \#\left(S^{1} \times S^{n-1}\right)$, and doing surgery on the circle of $S^{1} \times S^{n-1}$ we get $S^{n}$. Hence the result of the two surgeries is $M \# S^{n}=M$.

When $n \geq 4$, this proves that the Yamabe invariant is also unchanged under 0-dimensional surgeries and, therefore, we have finished the proof of Theorem 1.

Remark. Clearly, the Yamabe invariant is unchanged under surgery on an $(n-1)$ sphere $S$, unless $S$ separates $M$.

Let $\widehat{M}=M \#\left(S^{k} \times S^{n-k}\right)$, where $M$ is a smooth connected compact manifold of dimension $n \geq 5$. Doing surgery on any of the spheres $S^{k}$ or $S^{n-k}$ in $\widehat{M}$ produces a manifold diffeomorphic to $M$. And, of course, either $k$ or $n-k$ is different from 1 and $n-2$ (note also that $M$ admits a metric of positive scalar curvature if and only if $\widehat{M}$ does). Therefore, it follows from Theorem 1 that,

Corollary 1. Let $M$ be any compact smooth connected manifold $M$ of dimension $n \geq 5$. Assume that the Yamabe invariant of $M$ is non-positive. Then $Y(M)=Y\left(M \#\left(S^{k} \times S^{n-k}\right)\right)$ for all $0 \leq k \leq n$.

The previous result is definitely not true in the 4-dimensional case. Recall that C.T.C. Wall proved in [14] that if two compact simply connected four manifolds $M^{1}$ and $M^{2}$ have isomorphic intersection forms, then for $l$ big enough the connected sum of $M^{1}$ with $l$ copies of $S^{2} \times S^{2}$ is diffeomorphic to the connected sum of $M^{2}$ with $l$ copies of $S^{2} \times S^{2}$. Suppose that we have a pair $M^{1}, M^{2}$ of 4-manifolds with isomorphic intersection forms such that $Y\left(M^{1}\right)<0$ while $Y\left(M^{2}\right)>0$. Then it follows from the previous comments (and the fact that the family of manifolds admitting positive scalar curvature metrics is closed under connected sums $[4,10])$, that for $l$ big enough, $Y\left(M^{1} \# l\left(S^{2} \times S^{2}\right)\right)>0$. For example, we can take $M^{1}$ to be a smooth hypersurface of odd degree $d \geq 5$ in $C P^{3}$. C. LeBrun proved in [7] that $Y\left(M^{1}\right)<0$, while it is well known, from Freedman's classification of compact simply connected topological four-manifolds, that $M^{1}$ is homeomorphic to a connected sum of copies of $C P^{2}$ (some of these copies taken with the reversed orientation); and such a connected sum has positive Yamabe invariant (since the Fubini-Study metric in $C P^{2}$ has positive scalar curvature).

Note that given a 4-manifold $M$ and any "trivial $S^{1}$ " in $M$ (i.e. a small, simple loop in a neighbourhood of a point), doing surgery on such a circle produces $M \#\left(S^{2} \times S^{2}\right)$. Hence we can see from the previous observation that Theorem 1 is close to optimal:

Proposition 2. There are examples of 4-manifolds $M$, with negative Yamabe invariant, on which one can strictly increase the Yamabe invariant by doing 
surgery on dimension 1. It follows that there are also examples where one can strictly decrease the invariant by doing surgery in dimension 2.

We can now give some new exact computations of the invariant in dimension 4. Using Seiberg-Witten techniques, C. LeBrun proved in [7] that if $X$ is a minimal compact complex surface of general type and $M$ is obtained by blowing up $X$ any number of times, then

$$
Y(M)=Y(X)=-4 \pi \sqrt{2 c_{1}^{2}(X)} .
$$

Note also that doing 0-dimensional surgery on a connected four-manifold $M$ produces a manifold diffeomorphic to $M \#\left(S^{1} \times S^{3}\right)$. Therefore, it follows from Theorem 1 that,

Proposition 3. Let $M$ be a compact four-dimensional manifold with non-positive Yamabe invariant. Then $Y(M)=Y\left(M \#\left(S^{1} \times S^{3}\right)\right)$.

Using the results of LeBrun mentioned previously we get new exact computations of the invariant:

Proposition 4. Let $X$ be a minimal compact complex surface of general type. Let $M$ be a manifold obtained by taking the connected sum of any number of copies of $S^{1} \times S^{3}$ with the blow-up of $X$ at any number of points. Then $Y(M)=$ $-4 \pi \sqrt{2 c_{1}^{2}(X)}$.

\section{Manifolds without Einstein metrics}

Finally, the results from the previous section produce numerous new examples of compact four-manifolds which do not admit any Einstein metric. Let $M$ be obtained by blowing up $l$ times the minimal compact complex surface of general type $X$. Let $N=N(k, l)$ be the manifold obtained as the connected sum of $M$ with $k$ copies of $S^{1} \times S^{3}$. Suppose that $N$ admits an Einstein metric $g$. Then it follows from the Gauss-Bonnet formula that

$$
2 \chi(N) \pm 3 \tau(N)=\frac{1}{4 \pi^{2}} \int_{N}\left(2\left|W_{ \pm}\right|^{2}+\left(s_{g}^{2} / 24\right)\right) d \operatorname{vol}_{g} .
$$

But,

$$
\begin{aligned}
\frac{1}{4 \pi^{2}} \int_{N}\left(2\left|W_{ \pm}\right|^{2}+\left(s_{g}^{2} / 24\right)\right) d \operatorname{vol}_{g} \geq \frac{1}{4 \pi^{2}} \int_{N} \frac{s_{g}^{2}}{24} d \operatorname{vol}_{g} \\
\geq \frac{1}{96 \pi^{2}}(Y(N))^{2}=\frac{1}{3} c_{1}^{2}(X),
\end{aligned}
$$

where the last inequality follows, because the Yamabe invariant of $N$ is the negative of the square root of the infimum over the space of all Riemannian metrics on $N$ of the $L^{2}$ norm of the scalar curvature (we have already mentioned this result in the introduction,) and the final equality is precisely Corollary 2. 
Hence, we have seen that if $N$ admits an Einstein metric, then

$$
2 \chi(N)-3|\tau(N)| \geq(1 / 3) c_{1}^{2}(X) .
$$

This implies in many cases that such an Einstein metric cannot exist.

For instance, let us now prove Theorem 2. Let $M$ be a (compact) complex hyperbolic surface. In this case, one knows that $\chi(M)=3 \tau(M)>0$, and if $N$ is obtained as the connected sum of $M$ and $k$ copies of $S^{1} \times S^{3}$, the above inequality reads: $3 \tau(M)-4 k \geq 3 \tau(M)$. Therefore, $N$ cannot admite any Einstein metric.

Remark. Note that the Hitchin-Thorpe inequality only assures that an Einstein metric does not exist on $M \# k\left(S^{1} \times S^{3}\right)$ when $k \geq(3 / 4) \tau(M)$.

For a more general case, let $X$ be a minimal compact complex surface of general type which has non-positive signature. As before, let $M$ be the blow up of $X$ at $l$ points and let $N$ be the connected sum of $M$ and $k$ copies of $S^{1} \times S^{3}$. Note that $M$ and $N$ also have non-positive signature. If $N$ admits an Einstein metric we get from the previous computations that $c_{1}^{2}(X)-l-4 k \geq(1 / 3) c_{1}^{2}(X)$. Therefore,

Theorem 3. If $k>(1 / 6) c_{1}^{2}(X)-l / 4$, then $N=N(k, l)$ does not admit an Einstein metric.

Remark. In this case the Hitchin-Thorpe inequality only assures the non-existence of an Einstein metric when $k \geq(1 / 4) c_{1}^{2}(X)-l / 4$.

\section{Acknowledgements}

The author would like to thank Claude LeBrun for his support and for numerous observations related to this work. He would also like to thank Vyacheslav Krushkal for many useful discussions, and the directors and staff of the MaxPlanck Institut for their hospitality during the preparation of this work.

\section{References}

[1] M. Anderson, Scalar curvature and geometrization conjectures for 3-manifolds, Comparison Geometry (Berkeley, CA, 1993-94), 49-82, Math. Sci. Res. Inst. Publ., 30, Cambridge Univ. Press, Cambridge, 1997.

[2] M. Anderson, Extrema of curvature functionals on the space of metrics on 3-manifolds, Calc. Var. Partial Differential Equations 5 (1997), 199-269.

[3] T. Aubin, Équations différentielles non linéaires et problème de Yamabe concernant la courbure scalaire, J. Math. Pures Appl. (9) 55 (1976), 269-296.

[4] M. Gromov and H.B. Lawson, The classification of simply connected manifolds of positive scalar curvature, Ann. of Math. (2) 111 (1980) 423-434.

[5] O. Kobayashi, Scalar curvature of a metric with unit volume, Math. Ann. 279 (1987), $253-265$.

[6] C. LeBrun, Kodaira dimension and the Yamabe problem, to appear in Comm. Anal. Geom.

[7] _ Four-Manifolds without Einstein metrics, Math. Res. Lett. 3 (1996), 133-147.

[8] J. Petean and G. Yun, Surgery and the Yamabe invariant, to appear in GAFA. 
[9] A. Sambusetti, An obstruction to the existence of Einstein metrics on 4-manifolds, C.R. Acad. Sci. Paris Sér. I Math. 322 (1996), 1213-1218.

[10] R. Schoen and S.-T. Yau, On the structure of manifolds with positive scalar curvature, Manuscripta Math. 28 (1979), 159-183.

[11] R. Schoen, Conformal deformation of a Riemannian metric to constant scalar curvature, J. Differential Geom. 20 (1984), 479-495.

[12] _ - Variational theory for the total scalar curvature functional for Riemannian metrics and related topics, Topics in calculus of variations (Montecatini Terme, 1987), 120154, Lecture Notes in Math. 1365, Springer-Verlag, Berlin-New York, 1989.

[13] N.S. Trudinger, Remarks concerning the conformal deformation of Riemannian structures on compact manifolds, Ann. Scuola Norm. Sup. Pisa (3) 22 (1968), 265-274.

[14] C.T.C. Wall, On simply connected 4-manifolds, J. London Math. Soc. 39 (1964), 141-149.

[15] H. Yamabe, On a deformation of Riemannian structures on compact manifolds, Osaka Math. J. 12 (1960), 21-37.

MaX-Planck Institut für Mathematik, Bonn, Germany

E-mail address: jimmy@fractal.cimat.mx 